\title{
Meta-analysis of expression and methylation signatures indicates a stress-related epigenetic mechanism in multiple neuropsychiatric disorders
}

\author{
Kaiyi Zhu (D) ${ }^{1,2}$, Tai-Hsien Ou Yang ${ }^{1,2,5}$, Vincent Dorie ${ }^{3}$, Tian Zheng ${ }^{3,4}$ and Dimitris Anastassiou , 2,3 $^{2}$
}

\begin{abstract}
Similar environmental risk factors have been implicated in different neuropsychiatric disorders (including major psychiatric and neurodegenerative diseases), indicating the existence of common epigenetic mechanisms underlying the pathogenesis shared by different illnesses. To investigate such commonality, we applied an unsupervised computational approach identifying several consensus co-expression and co-methylation signatures from a data cohort of postmortem prefrontal cortex (PFC) samples from individuals with six different neuropsychiatric disordersschizophrenia, bipolar disorder, major depression, alcoholism, Alzheimer's and Parkinson's—as well as healthy controls. Among our results, we identified a pair of strongly interrelated co-expression and co-methylation (E-M) signatures showing consistent and significant disease association in multiple types of disorders. This E-M signature was enriched for interneuron markers, and we further demonstrated that it is unlikely for this enrichment to be due to varying subpopulation abundance of normal interneurons across samples. Moreover, gene set enrichment analysis revealed overrepresentation of stress-related biological processes in this E-M signature. Our integrative analysis of expression and methylation profiles, therefore, suggests a stress-related epigenetic mechanism in the brain, which could be associated with the pathogenesis of multiple neuropsychiatric diseases.
\end{abstract}

\section{Introduction}

Major psychiatric disorders, such as schizophrenia and bipolar disorder, and neurodegenerative diseases, such as Alzheimer's and Parkinson's, are all pathologically related to abnormalities in the brain ${ }^{1-4}$ with different manifestations in each case, but the underlying etiologies remain largely elusive. Extensive research has implicated environmental factors in the pathogenesis of such neuropsychiatric disorders ${ }^{5-9}$, operating through epigenetic mechanisms to change gene expression and thereby

Correspondence: Dimitris Anastassiou (d.anastassiou@columbia.edu)

'Department of Systems Biology, Columbia University, New York, NY 10032 USA

${ }^{2}$ Department of Electrical Engineering, Columbia University, New York, NY 10027, USA

Full list of author information is available at the end of the article. disrupting particular biological functions in brains. For example, in the case of several types of dementia, including Alzheimer's disease, environmental influences have been associated with the risk of disorder, which can lead to epigenetic transformations, such as altering DNA methylation and histone modification, over time ${ }^{10}$. Furthermore, striking experimental evidence connecting environmental stress and pathogenic outcome has been provided by research on both animals ${ }^{11-13}$ (rodents and primates) and humans ${ }^{14,15}$ (monozygotic twins with discordant disease states). The LEARn (latent early-life associated regulation) model was proposed ${ }^{16}$ as an epigenetic explanation for neurobiological disorders. On the other hand, it has been shown that transcriptomes and DNA methylation patterns of different brain regions differ substantially ${ }^{17,18}$ across brain regions. In this study, we

\section{(c) The Author(s) 2019}

(c) (i) Open Access This article is licensed under a Creative Commons Attribution 4.0 International License, which permits use, sharing, adaptation, distribution and reproduction cc) in any medium or format, as long as you give appropriate credit to the original author(s) and the source, provide a link to the Creative Commons license, and indicate if changes were made. The images or other third party material in this article are included in the article's Creative Commons license, unless indicated otherwise in a credit line to the material. If material is not included in the article's Creative Commons license and your intended use is not permitted by statutory regulation or exceeds the permitted use, you will need to obtain permission directly from the copyright holder. To view a copy of this license, visit http://creativecommons.org/licenses/by/4.0/. 
focused on one specific brain region, the prefrontal cortex (PFC), which has been implicated in the etiology of different neuropsychiatric diseases ${ }^{19,20}$.

We reasoned that the co-expression and co-methylation modules can be used as signatures to represent some particular biomolecular events, and that the modules shared by different diseases indicate common mechanisms. Moreover, analyzing the interrelationships between signatures can help in further understanding such mechanisms. For example, a significant association observed between some co-expression and co-methylation signatures can indicate a particular epigenetic regulation.

For that purpose, we first assembled multiple publicly available gene expression and DNA methylation data sets obtained from postmortem adult PFC samples across six different neuropsychiatric disorders along with healthy controls. We then identified a number of "consensus" PFC co-expression and co-methylation signatures, present in similar forms across multiple data sets, using an unsupervised methodology ${ }^{21}$. Integrative analysis of these signatures along with supervised analysis of available phenotypic associations suggested that a particular epigenetic abnormality could be involved in the pathogenesis of different neuropsychiatric illnesses.

\section{Materials and methods}

\section{Data sets and preprocessing}

Our discovery data cohorts consist of publicly available expression data and DNA methylation data of postmortem PFC samples from 426 subjects (242 cases, 184 controls) and 823 subjects (406 cases, 417 controls), respectively. The details of sample information can be found in Table 1. Most of the publicly available human postmortem PFC samples were obtained using the Affymetrix Human Genome U133 array and the Illumina HumanMethylation 450k beadchip for expression and DNA methylation, respectively. Because our multi-dataset algorithmic implementation works best with uniform data properties, we restricted the data sets to those types of profiling platform. We eliminated from consideration data sets representing repeated runs of the samples from the same subjects, thus avoiding replicates in the data cohorts that we collected for consensus signature identification, which could have otherwise biased the results. We also required that the number of either cases or controls should be at least 10 for expression data and 15 for methylation data (because of the larger number of probes for the methylation platform).

We downloaded the raw data sets and preprocessed them as follows. Data sets with gene-expression values were profiled using either of two Affymetrix platforms, but we only used HG-U133A probes for analysis in this study so that every individual expression data set contains the same probe set. The raw CEL files were log- transformed and RMA normalized for each individual data set with default settings as implemented in the $R$ Bioconductor affy package ${ }^{22}$. For DNA methylation analysis, we obtained $\beta$ values from the methylated and unmethylated signal intensities for each individual data set by using the dasen function in the $\mathrm{R}$ Bioconductor wateRmelon package ${ }^{23}$.

The validation data sets used in this study include additional microarray and RNA-seq data sets. The microarray data sets include GSE36980 for Alzheimer's disease $^{24}$ and GSE49376 for Alcoholism ${ }^{25}$, which were not included in the consensus analysis because they were profiled on different platforms. We normalized them in the same way as we did for the Affymetrix microarray data. The RNA-seq data sets, covering four out of the six neuropsychiatric diseases, include PFC samples from GSE68719 for Parkinson's ${ }^{26}$, GSE101521 for major depression $^{27}$, bipolar disorder and schizophrenia samples as part of the BrainGVEX study (available on Synapse with accession number syn4590909) within the PsychENCODE Consortium ${ }^{28}$. For RNA-seq data sets deposited on Gene Expression Omnibus (GEO), we normalized the raw counts individually using DESeq $2^{29}$, removed genes whose expression values were zero in more than half of the samples, and then performed $\log 2$-transformation. For the BrainGVEX data, we downloaded the normalized version from https://github. $\mathrm{com} / \mathrm{mgandal} /$ Shared-molecular-neuropathology-acrossmajor-psychiatric-disorders-parallels-polygenic-overlap/ tree/master/working_data/RNAseq.

\section{Attractor-finding algorithm \\ General version for individual data sets}

The attractor-finding algorithm is an unsupervised method for identifying signatures of mutually associated features from a matrix containing values of features (rows) in different samples (columns). Therefore, using expression or methylation matrices, it identifies coexpression and co-methylation signatures, respectively.

The details of the general attractor-finding algorithm can be found in our previous work ${ }^{21,30}$. Briefly, the algorithm uses an iterative procedure to collect mutually associated features, converging to the core ("heart") of the underlying co-expression or co-methylation mechanism. The association measure we used is based on the mutual information $(\mathrm{MI})^{31}$, which generally captures even nonlinear relationships between variables. To outline the process in the case of gene expression data, it starts from a "seed" (e.g., the expression of one particular gene). In the first iteration, all genes are ranked in terms of their MI with the seed gene, and a "metagene" is created as a hypothetical gene whose expression values, for each sample, are equal to the weighted average of the expression values of all genes, where each weight is defined as a 
Table 1 Description of data sets assembled for consensus analysis. (a) Gene expression cohorts. (b) DNA methylation cohorts

\begin{tabular}{lllll}
\hline (a) & & & \\
Data set & Disease & Platform & Brain region & Sample size (control:case) \\
\hline Ryan et al. ${ }^{55}$ [GSE5388] & Bipolar disorder & HG-U133A & BA9 & $31: 30$ \\
Maycox et al. ${ }^{56}$ [GSE17612] & Schizophrenia & HG-U133-P2 & BA10 & $23: 28$ \\
Zhang et al. ${ }^{57}$ Z Zheng et al. ${ }^{58}$ [GSE20168] & Parkinson's & HG-U133A & BA9 & $15: 14$ \\
Narayan et al. ${ }^{59}$ [GSE21138] & Schizophrenia & HG-U133-P2 & BA46 & $29: 30$ \\
SMRI AltarC & Multiple ${ }^{\text {a }}$ & HG-U133A & BA46/10 & $11: 33$ \\
SMRI Bahn & Multiple & BA46 & $33: 65$ \\
Chang et al. ${ }^{60}$ [GSE54567/54568/54570] & Major depression & HG-U133A & BA9 & $42: 42$
\end{tabular}

(b)

\begin{tabular}{|c|c|c|c|c|}
\hline Data set & Disease & Platform & Brain region & Sample size (control:case) \\
\hline Xu et al..$^{25}$ [GSE49393] & Alcoholism & HM450 & BA9 & $23: 23$ \\
\hline Lunnon et al..$^{61}$ [GSE59685] & Alzheimer's & HM450 & PFC & $24: 56$ \\
\hline Wockner et al. ${ }^{62}$ [GSE61107] & Schizophrenia & HM450 & PFC & $24: 24$ \\
\hline Pidsley et al.. ${ }^{63}$ [GSE61380/61431] & Schizophrenia & HM450 & BA9 & $38: 38$ \\
\hline Jaffe et al. ${ }^{64}$ [GSE74193] & Schizophrenia & HM450 & BA46/9 & 240:191 \\
\hline Smith et al. ${ }^{65}$ [GSE80970] & Alzheimer's & HM450 & PFC & $68: 74$ \\
\hline
\end{tabular}

BA Brodmann area, HM450 Illumina Infinium Human Methylation 450 Beadchip, PFC prefrontal cortex (for which Brodmann areas not specified), HG-U133A Affymetrix Human Genome U133A Array, HG-U133-P2 Affymetrix Human Genome U133 Plus 2.0 Array, SMRI Stanley Medical Research Institute

a. Cases consist of 11 bipolar disorder, 11 major depressive disorder, and 11 schizophrenia patients

b. Cases consist of 31 bipolar disorder and 34 schizophrenia patients

function of the MI of that gene with the seed gene. Each subsequent iteration updates the metagene, so that the weight of each gene in the new metagene is defined as a function of the MI of that gene with the previous metagene. The process is repeated until convergence to an "attractor metagene". From the attractor metagene, we can extract the top-ranked genes (those with the highest weights), together with a "score" (ranging from 0 to 1 ) for each of these genes, which measures the "strength" of the membership of that gene in the signature. If the strength of, say, the 10th ranked gene is $>0.5$, this suggests that there is a strong co-expression involving at least ten genes, and that the genes with the highest scores in the attractor metagene point to the core of the biological mechanism underlying that co-expression. The same attractor algorithm can be applied for other types of mutually correlated features, rather than genes. Therefore, more generally, the term "metagene" is an example of a "metafeature", and it has also been implemented in MATLAB's metafeatures function in the Bioinformatics Toolbox.

Using every available gene as seed identifies a limited number of strong co-expression signatures, each resulting in identical form from numerous seed genes. For methylation data, due to the excessively high number of methylation probes, we used a heuristic procedure for the exhaustive search to reduce computational complexity. The procedure, together with additional selection and filtering criteria for the validity of converged signatures to represent significant biological events (such as having a sufficient number of genes with high scores in each of them) are detailed in www.synapse.org/\#!Synapse: syn 5909000 .

\section{Probe-selection version for data sets with multiple probes for same gene}

Different platforms have different probe designs and sometimes each gene may have multiple measurements at different probes, which are often highly correlated with each other, rather than representing independent gene isoforms. This can create a bias of favoring genes with multiple probes. To avoid this kind of bias, we analyzed the gene expression and methylation data sets by using a "probe-selection" version of the attractor-finding algorithm. As in the general algorithm, the probe-selection algorithm computes the association between the metafeature and all the available probes. It has an additional step, however, in which for each feature it only selects one 
probe having the highest weight. Only those probes are used for updating the metafeature for the next iteration. Due to the nature of the probe-selection algorithm, probes not associated with unique genes are ignored.

\section{Consensus version for multiple data sets}

To identify common signatures shared across multiple diseases, we used a "consensus" version of the attractorfinding algorithm, which simultaneously takes into account all individual data sets. In each iteration for generating a new metafeature, the association measure of each feature with the immediately preceding metafeature is evaluated as the weighted median of the corresponding association measures taken from the individual data sets. The weights are proportional to the number of samples included in each individual data set. In one particular case, because the sample size of one methylation data set (GSE74193) is one magnitude higher than those of the other methylation data sets, we divided it into smaller subsets based on samples' processing plates, resulting in eleven individual methylation data sets used in final consensus analysis.

Both the above probe-selection and consensus methods of the attractor-finding algorithm are also detailed in www.synapse.org/\#!Synapse:syn5909000.

\section{Filtering consensus signatures by analyzing their presence in individual data sets}

After identifying the consensus signatures, for each data set, we used the average value (expression or methylation) of the top ten probes of all the consensus signatures as seeds to run the probe-selection attractor-finding algorithm, thus deriving the particular individual versions of each signature. Then, we evaluated the pairwise overlap between each individual signature and each consensus signature in terms of gene symbols using the hypergeometric test (one-tailed version of Fisher's exact test). We accepted the presence of a consensus signature in the individual data set if its overlap with the individual signature that it derived was the most significant (i.e., with the smallest $P$ value) compared with its overlaps with other individual signatures of this data set and had $P$ value less than 0.05. After obtaining the results for all the individual data sets, we removed from the final list any consensus signatures that were not present in the majority (i.e., more than half) of the individual data sets.

\section{Statistical analysis}

As described above, each attractor signature defines a ranked set of genes along with selected probes depending on their scores. The average values of the top ten probes were used to represent the levels of corresponding signatures. For DNA methylation profiles, we transformed the methylation to $M$ values as recommended ${ }^{32}$ and then took the average.

\section{Cell type specificity analysis}

The significance of cell type enrichment was assessed with the hypergeometric test by comparing the cell type markers and the genes for which the mapped probes have scores higher than 0.5 in the signature. To correct for multiple testing, we adjusted the resulting $P$ values with the false discovery rate (FDR) method using the p.adjust $\mathrm{R}$ function with parameter method $=$ "fdr". A signature is considered to be enriched for one specific cell type if it has significant overlap $(P<0.05)$ with each reference list of markers.

\section{Functional enrichment analysis}

We used the Molecular Signatures Database (MSigDB) with the gene set enrichment analysis (GSEA) software ${ }^{33}$ (v6.2) to explore the biological functions or processes overrepresented in specific gene sets, such as the identified co-expression and co-methylation signatures. The MSigDB database contains eight major annotated gene set collections, including Gene Ontology (GO) gene sets, hallmark gene sets, etc. It outputs the hypergeometric $P$ value and the FDR $q$ value according to the Benjamini-Hochberg procedure as an estimate of statistical significance for the overlap with these gene sets. To provide evidence of translational impact, we used the STRING $^{34}$ database (v10.5) to investigate the protein-protein interactions (PPIs) and protein functional analysis within each signature. For each signature, we used genes with scores higher than 0.5 as inputs, and limited their size to 500. FDR $q$ values $<0.05$ were considered significant.

\section{Association identification}

To investigate the association of the signatures with disease diagnosis, we used the linear mixed-effects (LME) model to evaluate the significance of disease association for each type of disorder. Since there are multiple data cohorts of the same disorder included, we used a random effect of study to consider the inter-study variability. To account for potential confounding effects, we evaluated results derived from two LME models, as follows:

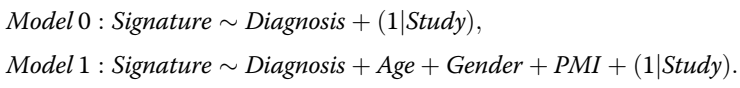

In Model 0, we obtained a "pure" significance of disease association without including other covariates, and in Model 1, we obtained a "confounder-adjusted" disease significance by treating age, gender, and postmortem interval (PMI), the covariates which are available in most of the data sets, as fixed effects. We used the lmer function implemented in the lme4 $\mathrm{R}$ package ${ }^{35}$ to fit the model 
using restricted maximum likelihood (REML, the default in lme4), and derived $P$ values by the Satterthwaite's degrees of freedom method with the lmerTest R package, as suggested for producing acceptable Type I error even for smaller sample sizes ${ }^{36}$. We further took into account the potential confounding effects of other covariates (antipsychotics dose, method of death, substance abuse, and smoking) which are only available in some data sets. We assessed their association with corresponding signatures separately in individual data set by performing one-way analysis of variance. We also performed the Mann-Whitney $U$ test, a nonparametric approach, to evaluate the disease association for each disease in the individual data sets to confirm the results. In all cases above, the threshold of statistical significance for $P$ values was set to 0.05 .

The correlation between same-type signatures (i.e., expression to expression, methylation to methylation) was examined using the Pearson's correlation test. The "expression-methylation" ("E-M") correlation between one co-expression signature and one co-methylation signature cannot be directly evaluated by using the same method, because there are no expression and methylation data coming from identical samples. As an alternative, we evaluated the E-M interrelationship through gene membership comparison by taking a significant overlap as indication that indeed methylation of the intersection genes affects the expression of those genes. We used the hypergeometric test to evaluate the significance of the overlap between the mapped gene symbols of the two signatures, for which the total gene pool was the intersection of the genes included in both expression and methylation platforms. The resulting $P$ values were adjusted by the FDR method. Similarly, all $P$ values for measuring the significance of overlaps in the context were evaluated using hypergeometric test.

\section{Results}

The consensus attractor finding (Materials and methods) of the combined data cohorts identified three consensus co-expression signatures and five consensus comethylation signatures, to which we refer as E1 through E3 and M1 through M5, respectively. The top-ranked probes and the mapped gene symbols of each signature are listed in Table 2. A more complete description of all the signatures can be found in Data Table S1.

Taking into consideration the gender-related differences in gene expression and methylation, including the fact that there are such differences in neuropsychiatric disorders $^{37}$, we first investigated if any signature is related

Table 2 Top probes in each PFC consensus signature. There are three columns shown for each signature, which denote the probe ID, gene symbols, and scores for the top 15 probes. (a) Consensus co-expression signatures, E1-E3. (b) Consensus co-methylation signatures, M1-M5

(a)

\begin{tabular}{|c|c|c|c|c|c|c|c|c|}
\hline \multicolumn{4}{|l|}{ E1 } & \multicolumn{3}{|l|}{ E2 } & \multicolumn{2}{|l|}{ E3 } \\
\hline Probes & Genes & Scores & Probes & Genes & Scores & Probes & Genes & Scores \\
\hline 209300_s_at & NECAP1 & 0.8463 & 202800_at & SLC1A3 & 0.8772 & 209769_s_at & SEPT5-GP1BB & 0.8030 \\
\hline 212990_at & SYNJ1 & 0.8265 & 207761_s_at & METTL7A & 0.8247 & 217696_at & FUT7 & 0.7826 \\
\hline 202854_at & HPRT1 & 0.8228 & 203908_at & SLC4A4 & 0.8194 & 214122_at & PDLIM7 & 0.7676 \\
\hline 208841_s_at & G3BP2 & 0.8092 & 202936_s_at & SOX9 & 0.8180 & 216940_x_at & YBX1 & 0.7666 \\
\hline 213745_at & ATRNL1 & 0.8031 & 201667_at & GJA1 & 0.8092 & 214105_at & sOCS3 & 0.7537 \\
\hline 204552_at & INPP4A & 0.8022 & 212230_at & PPAP2B & 0.7954 & 209979_at & ADARB1 & 0.7524 \\
\hline 201889_at & FAM3C & 0.8009 & 201876_at & PON2 & 0.7926 & 209730_at & SEMA3F & 0.7439 \\
\hline 205352_at & SERPINI1 & 0.8005 & 212377_s_at & $\mathrm{NOTCH} 2$ & 0.7898 & 216680_s_at & EPHB4 & 0.7425 \\
\hline 205280_at & GLRB & 0.7963 & 203296_s_at & ATP1A2 & 0.7888 & 207306_at & TCF15 & 0.7417 \\
\hline 209274_s_at & ISCA1 & 0.7942 & 206465_at & ACSBG1 & 0.7684 & 216345_at & ZSWIM8 & 0.7385 \\
\hline 211763_s_at & UBE2B & 0.7915 & 209210_s_at & FERMT2 & 0.7624 & 206824_at & CES1P1 & 0.7325 \\
\hline 202670_at & MAP2K1 & 0.7909 & 221796_at & NTRK2 & 0.7613 & 216821_at & KRT8P11 & 0.7305 \\
\hline 218042_at & COPS4 & 0.7866 & 212850_s_at & LRP4 & 0.7505 & 202828_s_at & MMP14 & 0.7244 \\
\hline 221207_s_at & NBEA & 0.7864 & 205328_at & CLDN10 & 0.7432 & 216076_at & L3MBTL1 & 0.7219 \\
\hline 213423_x_at & TUSC3 & 0.7861 & 203120_at & TP53BP2 & 0.7412 & 205212_s_at & ACAP1 & 0.7214 \\
\hline
\end{tabular}




\begin{tabular}{|c|c|c|c|c|c|c|c|c|c|}
\hline \multicolumn{5}{|l|}{ (b) } & \multicolumn{3}{|c|}{ 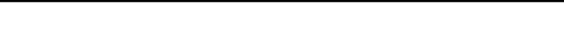 } & \\
\hline \multicolumn{5}{|l|}{ M1 } & \multicolumn{3}{|l|}{ M2 } & \multicolumn{2}{|l|}{ M3 } \\
\hline Probes & Genes & Scores & & Probes & Genes & Scores & Probes & Genes & Scores \\
\hline cg10717149 & SLC25A14 & 0.9911 & & cg22655232 & PPP1R2P9 & 0.9784 & cg26765599 & NMRAL1 & 0.9040 \\
\hline cg04317640 & SLC16A2 & 0.9910 & & cg11049634 & BCOR & 0.9778 & cg06081917 & BFAR & 0.9013 \\
\hline cg16221895 & EDA & 0.9908 & & cg05130312 & LOC286467 & 0.9740 & cg17032990 & MAP4K4 & 0.8983 \\
\hline cg14191108 & MAOA & 0.9907 & & cg14372935 & PIR & 0.9722 & cg02473439 & CCAR1 & 0.8979 \\
\hline cg10981178 & ZBTB33 & 0.9899 & & cg06780606 & EDA & 0.9716 & cg02193425 & FAM50B & 0.8972 \\
\hline cg26505478 & CUL4B & 0.9893 & & cg09791535 & GPC4 & 0.9714 & cg02313013 & TMCC3 & 0.8942 \\
\hline cg23696472 & TSPYL2 & 0.9892 & & cg09192294 & LAS1L & 0.9701 & cg00489902 & POLE & 0.8926 \\
\hline cg05806018 & AFF2 & 0.9890 & & cg07801607 & ZMAT1 & 0.9696 & cg10521450 & SH3PXD2A & 0.8922 \\
\hline cg11594566 & LINC00086 & 0.9884 & & cg04690567 & PHF8 & 0.9683 & cg02041593 & SEMA5B & 0.8917 \\
\hline cg10201390 & DYNLT3 & 0.9882 & & cg00098732 & HS6ST2 & 0.9662 & cg00245075 & GALNT6 & 0.8911 \\
\hline cg20749341 & LONRF3 & 0.9881 & & $\operatorname{cg} 12653510$ & XIST & 0.9662 & cg19885979 & TRIM26 & 0.8902 \\
\hline cg22164912 & GNL3L & 0.9877 & & cg27551771 & KIAA1210 & 0.9639 & cg00452755 & RCC1 & 0.8900 \\
\hline cg20766178 & NHSL2 & 0.9875 & & cg01037726 & PNCK & 0.9627 & cg02450267 & MOG & 0.8876 \\
\hline cg18989810 & DUSP9 & 0.9874 & & cg04704683 & POF1B & 0.9617 & cg23384027 & NFE2 & 0.8875 \\
\hline cg22604777 & MAGEH1 & 0.9874 & & cg08209935 & ARMCX5 & 0.9602 & cg02713352 & B4GALNT1 & 0.8874 \\
\hline \multicolumn{4}{|l|}{ M4 } & \multicolumn{4}{|l|}{ M5 } & & \\
\hline Probes & \multicolumn{2}{|l|}{ Genes } & Scores & \multicolumn{2}{|c|}{ Probes } & Genes & Scores & & \\
\hline cg12268888 & \multicolumn{2}{|l|}{ FAM198A } & 0.8628 & \multicolumn{2}{|c|}{ cg12547839 } & UBE2O & 0.8539 & & \\
\hline cg09063372 & \multicolumn{2}{|l|}{ HDGF } & 0.8506 & \multicolumn{2}{|c|}{ cg22330763 } & SLC29A1 & 0.8421 & & \\
\hline cg11150308 & \multicolumn{2}{|l|}{ SRP68 } & 0.8505 & \multicolumn{2}{|c|}{ cg04101806 } & AFF3 & 0.8203 & & \\
\hline cg16129988 & \multicolumn{2}{|l|}{ UQCRC1 } & 0.8468 & \multicolumn{2}{|c|}{ cg23400122 } & MSRA & 0.8179 & & \\
\hline cg11371394 & \multicolumn{2}{|l|}{ TGFBRAP1 } & 0.8406 & \multicolumn{2}{|c|}{ cg26218110 } & BAHCC1 & 0.8153 & & \\
\hline cg04233747 & \multicolumn{2}{|l|}{ PRELID2 } & 0.8403 & \multicolumn{2}{|c|}{ cg25119743 } & CELF2 & 0.8023 & & \\
\hline cg03330867 & \multicolumn{2}{|l|}{ TELO2 } & 0.8398 & \multicolumn{2}{|c|}{ cg06372223 } & SLC7A5 & 0.8023 & & \\
\hline cg04426297 & \multicolumn{2}{|l|}{ B3GAT3 } & 0.8394 & \multicolumn{2}{|c|}{ cg24897320 } & CYB561D1 & 0.8008 & & \\
\hline cg24695828 & \multicolumn{2}{|l|}{ ZNF566 } & 0.8324 & $\mathrm{cg} 1$ & 6739 & DMTN & 0.7987 & & \\
\hline cg01923255 & ATG14 & & 0.8321 & $\mathrm{cgO}$ & 2720 & PER2 & 0.7983 & & \\
\hline cg26897054 & DEDD2 & & 0.8317 & $\mathrm{cg} 1$ & 8776 & PACSIN1 & 0.7970 & & \\
\hline cg11111696 & ZNF438 & & 0.8312 & $\operatorname{cg} 2$ & 8252 & MSI2 & 0.7966 & & \\
\hline $\operatorname{cg} 25426560$ & DHX16 & & 0.8309 & $\mathrm{cg} 2$ & 7728 & LRP8 & 0.7962 & & \\
\hline cg05623562 & RBFA & & 0.8300 & $\mathrm{cgO}$ & 6743 & NTM & 0.7943 & & \\
\hline cg24715473 & CNEP1R1 & & 0.8290 & $\mathrm{cg} 2$ & 5981 & MEGF8 & 0.7929 & & \\
\hline
\end{tabular}

to gender. Among the co-methylation signatures, we found that M1 and M2 are purely gender-related (due to the presence of both genders in the data) (Fig. 1), consistent with the fact that their top-ranked genes are almost exclusively located in sex chromosomes. For example, XIST, one of the top genes of signature M2 (Table 2b), plays a critical role in the process of $\mathrm{X}$-chromosome inactivation in mammalian females, an early 


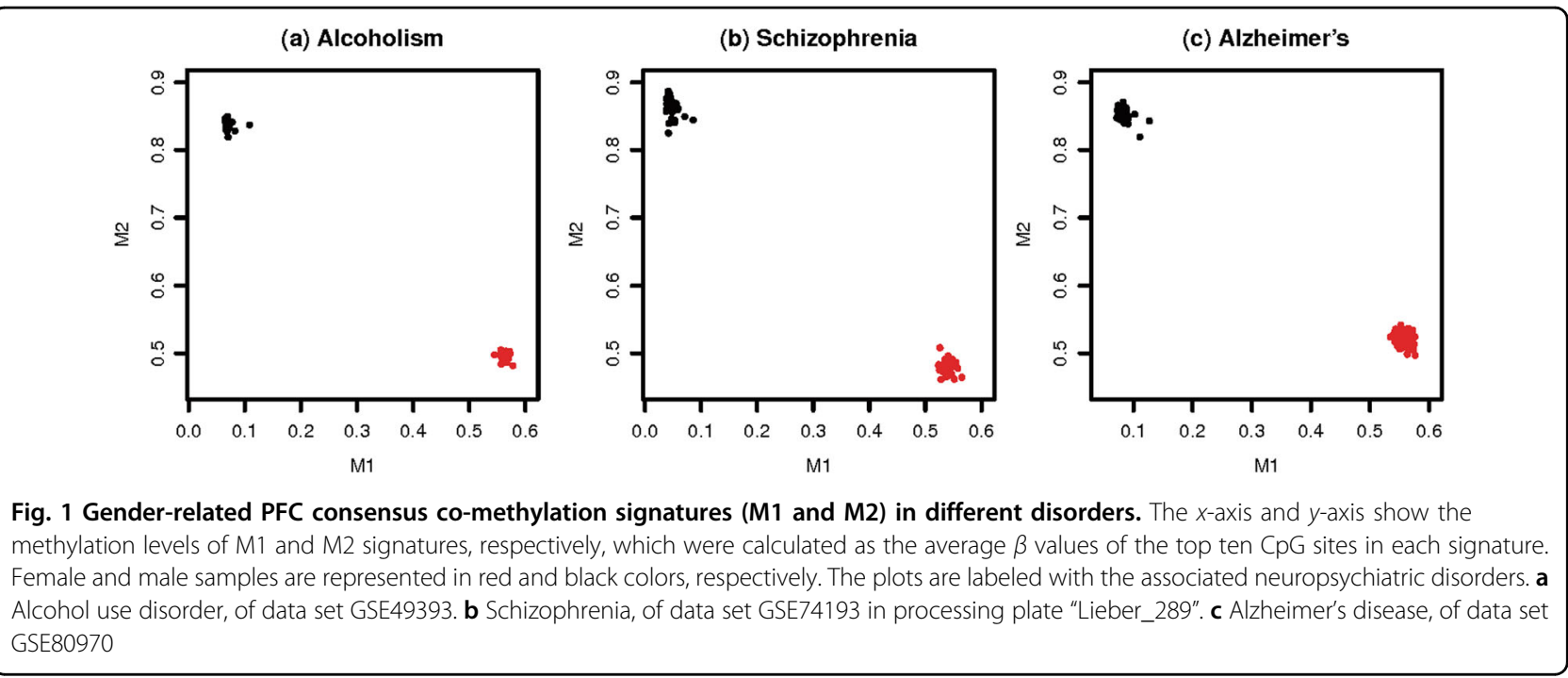

developmental mechanism through which one of the $\mathrm{X}$ chromosomes is silenced by the combination of DNA methylation and histone modifications to provide dosage compensation $^{38}$. Since they are otherwise unrelated with disease phenotypes, we do not include them in the following analyses.

Co-expression and co-methylation signatures include contributions from several distinct cell subpopulations in the heterogeneous brain tissues, from cell types such as neurons, astrocytes, oligodendrocytes, and immune cells, which can be further decomposed into cell subtypes. Because their corresponding probes have the property that their expression or methylation values tend to have higher or lower values in concordance, it is likely that some of these signatures reflect the relative abundance of such a subpopulation, which varies from sample to sample. Alternatively, co-expression or co-methylation may be due to the varying activation of a particular mechanism within the same subpopulation, as may result, e.g., when the expression levels of multiple genes are affected by their simultaneous methylation.

Therefore, we investigated which among the consensus signatures we found are predominantly due to the varying abundances of particular cell types across the samples. To identify the enrichment of the consensus signatures in cell-type-specific genes, we made use of two published gene lists of such gene markers derived from single cell analyses for reference ${ }^{39,40}$ (Materials and methods). The first reference list is taken from a study providing a classification of human brain cells into six major types ${ }^{39}$. The second reference list comes from a study providing a full list of marker genes of nine cell types found in the mouse corte ${ }^{40}$. Although not resulting from human tissues, these extensive and detailed listings from mice are useful for additional scrutiny and validation. The results of enrichment analysis of cell type markers using the two lists were highly consistent (Data Table S2). All of the signatures were found enriched for some specific cell types except for E3 and M4.

For the co-expression signatures, this analysis revealed that signature E1 is enriched for neuronal markers $(P=$ 0.0033 using the human markers). As the mouse cortex gene set contains particular neuronal subtypes, we further found that E1 has the highest overrepresentation of markers for the interneuron subtype $(P=0.0018)$. On the other hand, we found that signature E2 is highly enriched for astrocyte-specific markers (such as SOX9, GJA1 ranked at the top) in both gene lists $\left(P=2.2 \times 10^{-13}\right.$ using the human markers and $P=1.4 \times 10^{-16}$ using the mouse markers).

For the co-methylation signatures, we found that signatures M3 and M5 are enriched for markers of glia and neurons, respectively (Data Table S2), and they were strongly and negatively correlated with each other (Pearson's $r<-0.75, P<1.2 \times 10^{-8}$; Data Table S3). We validated this finding by checking the methylation levels of M3 and M5 in an independent human PFC methylation data set of isolated neurons and nonneurons as well as manually mixed and bulk samples ${ }^{41}$ (GSE41826) (Fig. 2). Moreover, M3 was found associated with the coexpression signature E2 $(P=0.010$; Materials and methods), which is consistent with the aforementioned facts that E2 is overrepresented in astrocyte markers and M3 is enriched for different subtypes of glial cell including astrocytes. Taken together, these findings indicate that M3 and M5 reflect the relative abundances of neurons vs. glial cells in samples, which are negatively associated, by including particular hyper- and hypo-methylated loci in these two subpopulations.

We evaluated the signatures' associations with disease diagnosis considering potential confounding effects (Materials and methods; Supplementary Notes). As a 
result, we observed that the co-expression signatures E1 and E2 showed concordantly significant associations in more than one type of neuropsychiatric disorders (Data Table S4), and these associations are unlikely to be spurious due to confounding factors. Moreover, both signatures were enriched for PPI networks $(P<1.0 \times$

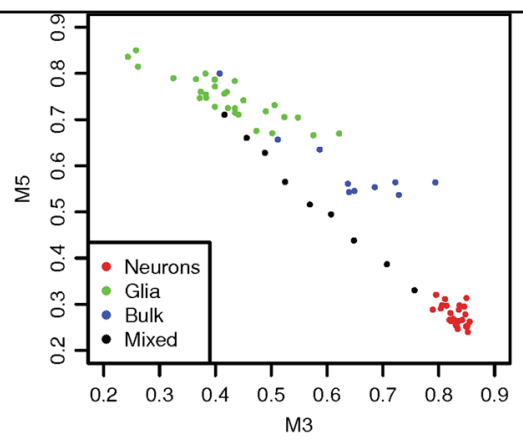

Fig. 2 Negative correlation between cell-type-specific PFC consensus co-methylation signatures (M3 and M5) in different cell populations. The $x$-axis and $y$-axis show the methylation levels of the M3 and M5 signatures, respectively, which were calculated as the average $\beta$ values of the top ten $\mathrm{CpG}$ sites in each signature. The samples come from the data set GSE41826, which includes

methylation data of separated neurons (red), separated glia (green), as well as mixed (black for manually mixed and blue for bulk samples) from healthy human PFC tissues. The proportion of neurons to nonneurons in the empirically mixed samples range from 10 to $90 \%$ in $10 \%$ increments
$10^{-16}$ for E1, $P=8.4 \times 10^{-14}$ for E2). Specifically, E1 was found broadly downregulated in several types of diseases, a topic discussed in detail in the next section.

The other signature, E2 was found upregulated in schizophrenia $\left(P=1.8 \times 10^{-3}\right)$ and bipolar disorder $(P=$ $\left.2.1 \times 10^{-3}\right)$. One of the GSEA top hits suggested that E2 is also enriched for genes which were found upregulated in brains with Alzheimer's disease ${ }^{42}$ (FDR $q$ value $=1.8 \times$ $\left.10^{-19}\right)$. Finally, we observed a substantial overlap between E2 and an astrocyte-related co-expression module (named "CD4") that was recently found positively associated with multiple psychiatric disorders ${ }^{43}\left(P=1.1 \times 10^{-114}\right)$. Functional annotation analysis indicated the enrichment for biological pathways related to nervous system development and glial cell differentiation.

\section{The E1-M4 pair indicates a disease-related stress- induced epigenetic mechanism}

The most significant E-M association was observed between signatures E1 and M4 $\left(P=1.9 \times 10^{-4}\right.$; Data Table S3), which share a large proportion of genes in common.

We first discuss the co-expression signature E1, which we found to be the one most significantly associated with disease diagnosis among all the identified consensus signatures. We observed significant downregulation of E1 in the presence of disease in several types of neuropsychiatric disorders except for depression and alcohol use

Table 3 Disease association of co-expression signature E1. Shown are the $\boldsymbol{P}$ values of association with diagnosis in different disorders resulting from the LME models (Materials and methods) for signature E1. Columns annotated by "Model 0 " and "Model 1", represent the "pure" and "confounder-adjusted" cases, respectively. Full results for other signatures can be found in Data Table S4

(a) Discovery data

Diseases

E1 (Model 0)

E1 (Model 1)

Schizophrenia

Bipolar disorder

$1.7 \times 10^{-3}$

$1.1 \times 10^{-3}$

$3.8 \times 10^{-5}$

$3.3 \times 10^{-5}$

Parkinson's

Major depression

0.013

0.040

0.19

0.13

(b) Validation data

Diseases

Bipolar disorder

0.014 
disorder, and we confirmed this striking association using validation data sets (Table 3). We took into account the potential confounding effects of all available traits (including age, gender, manner of death, substance abuse, antipsychotic treatment usage, etc.), and our results suggest that the significant disease association we identified in signature E1 may not be confounded by these factors (Materials and methods; Supplementary Notes).

The disease-associated downregulation of signature E1 is supported by its large overlap (using the genes for which the scores in E1 are higher than 0.5) with previously identified differentially expressed gene sets in different illnesses. For instance, E1 genes are overrepresented in a set of genes that were identified downregulated in the PFC of patients with schizophrenia ${ }^{44}\left(P=9.1 \times 10^{-7}\right)$. Furthermore, the top GSEA result for E1 revealed significant enrichment for genes downregulated in the brain from patients with Alzheimer's disease ${ }^{42}$ (FDR $q$ value $=3.0 \times$ $\left.10^{-317}\right)$. Finally, many common genes were found included in both E1 and a neuronal module (named "CD13") that had been found downregulated in multiple psychiatric illnesses ${ }^{43}\left(P=2.3 \times 10^{-40}\right)$. These findings strongly suggest that the downregulation of the consensus coexpression signature E1 represents an important biological event occurring in the brain affecting different neuropsychiatric disorders.

As previous studies indicated ${ }^{42,44}$, and in concordance with the GSEA results of signature E1, some of the downregulated genes are involved in biological processes related to neuronal functions including neurotransmitter transport, signaling pathways and various energy metabolism processes. On the other hand, as mentioned previously, E1 was found enriched in neuronal markers, particularly of the interneuron subtype, which allows for a possibility that the disease correlation of $\mathrm{E} 1$ is caused by the variances of this particular cell population, as was the case for signature E2. To elucidate in what ways E1 is related to interneurons and associated with disease, we designed an experiment as described below.

In the experiment, we made use of the set of interneuron density markers whose expression levels were identified to be significantly and positively correlated with the density of CALB1-positive GABAergic interneurons ${ }^{45}$, which also appeared as the second top GSEA hit for signature E1 (FDR $q$ value $=5.9 \times 10^{-250}$ ). The null hypothesis was that the downregulation of E1 observed in patients with disease is caused by the decreasing population of interneurons, in which case we should expect to see that the disease association becomes reduced when we remove from the E1 gene list the markers for interneuron density. Therefore, we compared the disease association of the subset of E1 genes without those interneuron markers, referred to as "Set E1/Interneuron" with that of the E1 signature. As a result, the disease association for
Table 4 Strengthened disease association compared with E1 alone. Shown are the confounder-adjusted $P$ values of association with diagnosis in different disorders for three E1-related gene sets in the discovery data. Columns "E1", "E1/Interneuron", and "E1 $\cap$ M4" represent the cases for E1 signature alone, the subset of E1 genes without GABAergic interneuron markers, and the intersection of genes included in both E1 and M4

\begin{tabular}{llll}
\hline Diseases & E1 & E1/interneuron & E1กM4 \\
\hline Schizophrenia & $1.1 \times 10^{-3}$ & $4.6 \times 10^{-4}$ & $2.5 \times 10^{-4}$ \\
Bipolar disorder & $3.3 \times 10^{-5}$ & $1.1 \times 10^{-5}$ & $2.7 \times 10^{-6}$ \\
Parkinson's & 0.040 & 0.086 & 0.032 \\
Major depression & 0.13 & 0.12 & 0.050 \\
\hline
\end{tabular}

the Set E1/Interneuron became stronger in most cases, rejecting the null hypothesis (Table 4, see columns named "E1" and "E1/Interneuron"). This suggests that the strong disease association of signature E1 in various neuropsychiatric disorders is not caused by the allocation of interneuron subpopulation in samples.

We then looked at the significant overlap of genes found between the co-expression signature E1 and the comethylation signature M4, which implies an underlying epigenetic regulation mechanism. We selected the overlapping genes between the E1 and M4 signatures (using

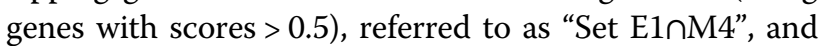
ranked them by the minimum of their scores in E1 and M4 gene lists so that the top gene has the highest minimum score (the ranked list of the top 15 genes of Set E1nM4 is shown in Table 5, while the full list can be found in Data Table S5). We evaluated the disease association of Set E1กM4 using the average expression values of the top-ranked ten genes in the set and compared with the case of E1 itself. As a result, we observed overall enhancement of the association with diagnosis in the cases of the Set E1 MM4 (Table 4, columns labeled "E1" and "E1กM4"). This result suggests that the comethylation signature M4 contributes to refining the coexpression signature E1 with respect to the association with disease diagnosis through an epigenetic mechanism.

Furthermore, regarding the co-methylation signature M4 itself, we found that it has a unique attribute among all the nongender-related consensus co-methylation signatures, in that it contains a remarkably high proportion of methylation probes located at promoter-associated regions $\left(P=8.0 \times 10^{-149}\right)$ and $C p G$ islands $(P=0)$ when compared with the overall sites for the methylation array (Data Table S6), implicating its function of epigenetic regulation.

To understand the biological functions represented by the E1-M4 signature, we applied functional annotation 
Table 5 Top-ranked genes in the Set E1nM4. This table shows the top 15 overlapping genes between signatures E1 and M4, ranked by the minimum of their scores in the two signature gene lists. The four columns represent the gene symbols, corresponding probe IDs in E1 and M4 signatures, and the minimum scores, respectively

\begin{tabular}{llll}
\hline Gene symbols & Probes in E1 & Probes in M4 & Min scores \\
\hline CAND1 & 208838_at & cg17524854 & 0.7499 \\
DYNC1LI1 & 217976_s_at & cg25390230 & 0.7253 \\
ATP5A1 & 213738_s_at & cg10619144 & 0.6912 \\
EFR3A & 212150_at & cg09396107 & 0.6786 \\
MEAF6 & 218165_at & cg03112782 & 0.6774 \\
PNMA1 & 218224_at & cg23681213 & 0.6772 \\
SEC23A & 212887_at & cg02056847 & 0.676 \\
ZNHIT3 & 212544_at & cg09922935 & 0.6697 \\
PPP2R5C & 201877_s_at & cg08393828 & 0.6676 \\
NDUFAB1 & 202077_at & cg21989500 & 0.6675 \\
EIF1B & 201738_at & cg25839330 & 0.6672 \\
PPP3CA & 202429_s_at & cg00302793 & 0.6667 \\
SLC30A9 & 202614_at & cg09414773 & 0.6599 \\
UQCRC2 & 212600_s_at & cg03031583 & 0.6595 \\
RGS7 & 206290_s_at & cg24472496 & 0.6592 \\
\hline
\end{tabular}

analysis on the intersection genes. First, significant enrichment of PPIs $\left(P=2.2 \times 10^{-16}\right)$ indicates the meaningful biological connections and regulatory functions among the proteins encoded by these genes. The small GTPase superfamily was the top hit by assessing the overlap with InterPro protein domains and features database ${ }^{46}$. Among the results of GSEA analysis, the set of genes downregulated in brains with Alzheimer's disease ${ }^{42}$ remains at the top $\left(\right.$ FDR $q$ value $=9.7 \times 10^{-115}$ ). Overrepresentation was also found in sets of genes having at least one occurrence of highly conserved motifs matching binding sites for transcription factors SP1 (FDR $q$ value $=$ $3.1 \times 10^{-25}$ ) and LEF1 (FDR $q$ value $=5.6 \times 10^{-14}$ ), which may provide hints about the nature of the underlying epigenetic mechanism. Moreover, we found that stressrelated biological processes (GO) were enriched in the genes of E1-M4 signature (FDR $q$ value $<10^{-6}$ ). We further confirmed that these stress-related gene sets were also overrepresented in the respective E1 and M4 signatures, but not in any of the other consensus signatures (Data Table S7).

Along with the significant $\mathrm{E}-\mathrm{M}$ interrelationship and strong disease association, these findings collectively suggest that the E1-M4 signature pair represents some stress-induced epigenetic mechanism, which could be associated with the underlying etiology of several neuropsychiatric disorders.

\section{Discussion}

To investigate the underlying pathological mechanism (s) common to various neuropsychiatric diseases, we did meta-analyses on the expression and methylation data of postmortem PFC samples collected from patients with six different neuropsychiatric disorders along with healthy controls (Table 1). By using our unsupervised approach, we identified several consensus co-expression and comethylation signatures present in similar forms across different data sets and diseases (Table 2). By scrutinizing these signatures' disease associations and interrelationships, our study revealed some biological abnormalities strongly associated with disease diagnosis.

For example, we identified an astrocyte-related coexpression signature, E2, which was observed upregulated in patients with schizophrenia and bipolar disorder, and functional enrichment analysis also indicated its overlap with genes found to be upregulated in Alzheimer's disease. Previous studies have suggested that the changes in expression of astrocyte markers could be linked to neuroinflammation in these diseases ${ }^{47,48}$. We did not observe such positive association in other disorders. Taking the major depressive disorder as an example, our results showed that, on the contrary, E2 is negatively, though not very significantly, associated with disease diagnosis (Data Table S4). Indeed, studies have reported persistent decreases in astrocyte-specific markers in patients with major depression ${ }^{49}$, indicating disease association with decreased density or hypofunction of astrocytes, and there is also experimental evidence provided for understanding the underlying pathogenic mechanism using animal models ${ }^{50}$.

Our work resulted in the derivation of several coexpression and co-methylation signatures using an algorithm designed to point to the core of the underlying mechanisms, which suggests that the top genes of such signatures are more biologically accurate compared with traditional clustering methods. However, the main feature of our study is the examination of the interrelationships between such expression and methylation signatures in search of epigenetic mechanisms. Understanding diseaseassociated epigenetic mechanisms may provide opportunities of developing novel therapeutic options.

Using this approach, our main finding was the novel discovery of a significant interrelationship between the co-expression signature E1 and co-methylation signature M4, which indicates an epigenetic relationship. On the one hand, the signature E1 is enriched for interneuron markers and we provided evidence that the derivation of the signature is due to a variation of the expression levels of such interneurons, rather than a varying abundance of 
their subpopulation. On the other hand, E1 is found strongly down regulated in multiple types of neuropsychiatric diseases (Table 3 ). The disease association becomes further strengthened when we consider the shared genes between E1 and M4 (Table 4), suggesting that this sharper disease-associated signature is related to an epigenetic mechanism involving the E1-M4 genes (Table 5). The overrepresentation of promoter-associated regions and $\mathrm{CpG}$ islands in the corresponding genes of M4 also complements its role of epigenetic regulation. Future experimental research on those genes has the potential of uncovering the details of the biological mechanism underlying the epigenetic signature and leading to therapeutic applications.

We did not, however, observe a significant disease association of the signature in major depressive disorder and in alcohol use disorder, suggesting that their diagnosis is often independent of the underlying biological mechanism. This is consistent with the fact that there is comorbidity between depression and alcohol use disorder $^{51}$. Such differences reflect the heterogeneity of neuropsychiatric disorders. For example, major depressive disorder is known to have small heritability compared with other disorders ${ }^{52}$.

In addition, functional annotation analysis of the E1-M4 genes revealed the enrichment of stress-related biological processes. While the genes highlighted under the identified GO terms may reflect internal cellular processes, most gene activities are attributed to pathways involving exogenous stressors. Notably, stress response and related epigenetic regulation mechanisms in the brain have been investigated and implicated in neuropsychiatric diseases ${ }^{8,9,53,54}$, bringing up the possibility that these effects are driven by psychiatry-relevant psychosocial stressors or other relevant biological processes and should be investigated in future studies.

Our findings should be interpreted in light of some caveats. Although we have taken care of controlling for all known confounders, unmeasured variables specific to disease states may influence the results to some degree in epigenomic and transcriptomic studies. The identified mechanisms in this study may well be causal and important underlying features to disease etiology, but there is also a chance that this is not true.

In summary, our integrative data mining and analysis of some of the identified consensus co-expression and comethylation signatures suggest the presence of a stressrelated epigenetic mechanism associated with different neuropsychiatric diseases.

\section{Author details}

'Department of Systems Biology, Columbia University, New York, NY 10032, USA. ${ }^{2}$ Department of Electrical Engineering, Columbia University, New York, NY 10027, USA. ${ }^{3}$ Data Science Institute, Columbia University, New York, NY 10027,
USA. ${ }^{4}$ Department of Statistics, Columbia University, New York, NY 10027, USA. ${ }^{5}$ Present address: Roche Innovation Center, New York, NY 10016, USA

Conflict of interest

The authors declare that they have no conflict of interest.

\section{Code availability}

The code for generating the signatures discussed in this study is available at https://github.com/zky0708/Meta-analysis-of-expression-and-methylationsignatures-in-neuropsychiatric-disorders-brains.

\section{Publisher's note}

Springer Nature remains neutral with regard to jurisdictional claims in published maps and institutional affiliations.

Supplementary Information accompanies this paper at (https://doi.org/ 10.1038/s41398-018-0358-5).

Received: 15 August 2018 Revised: 12 November 2018 Accepted: 9 December 2018

Published online: 22 January 2019

\section{References}

1. Craddock, N., O'Donovan, M. C. \& Owen, M. J. Genes for schizophrenia and bipolar disorder? Implications for psychiatric nosology. Schizophr. Bull. 32, 9-16 (2006).

2. Aarsland, D., Marsh, L. \& Schrag, A. Neuropsychiatric symptoms in Parkinson's disease. Mov. Disord. 24, 2175-2186 (2009).

3. Staal, W. G. et al. Structural brain abnormalities in patients with schizophrenia and their healthy siblings. Am. J. Psychiatry 157, 416-421 (2000).

4. Lyketsos, C. G. et al. Neuropsychiatric symptoms in Alzheimer's disease. Alzheimers Dement. 7, 532-539 (2011).

5. Migliore, L. \& Coppede, F. Genetics, environmental factors and the emerging role of epigenetics in neurodegenerative diseases. Mutat. Res. 667, 82-97 (2009).

6. Schuckit, M. A. Alcohol-use disorders. Lancet 373, 492-501 (2009).

7. Zucchi, F. C. et al. Maternal stress induces epigenetic signatures of psychiatric and neurological diseases in the offspring. PLoS One 8, e56967 (2013).

8. Klengel, T. \& Binder, E. B. Epigenetics of stress-related psychiatric disorders and gene $\times$ environment interactions. Neuron 86, 1343-1357 (2015).

9. Zannas, A. S. \& Chrousos, G. P. Epigenetic programming by stress and glucocorticoids along the human lifespan. Mol. Psychiatry 22, 640-646 (2017).

10. Maloney, B. \& Lahiri, D. K. Epigenetics of dementia: understanding the disease as a transformation rather than a state. Lancet Neurol. 15, 760-774 (2016).

11. Basha, M. R. et al. The fetal basis of amyloidogenesis: exposure to lead and latent overexpression of amyloid precursor protein and beta-amyloid in the aging brain. J. Neurosci. 25, 823-829 (2005).

12. $\mathrm{Wu}$, J. et al. Alzheimer's disease (AD)-like pathology in aged monkeys after infantile exposure to environmental metal lead (Pb): evidence for a developmental origin and environmental link for AD. J. Neurosci. 28, 3-9 (2008).

13. Dosunmu, R. et al. Lifespan profiles of Alzheimer's disease-associated genes and products in monkeys and mice. J. Alzheimers Dis. 18, 211-230 (2009).

14. Mastroeni, D., McKee, A., Grover, A., Rogers, J. \& Coleman, P. D. Epigenetic differences in cortical neurons from a pair of monozygotic twins discordant for Alzheimer's disease. PLoS One 4, e6617 (2009).

15. Dempster, E. L. et al. Disease-associated epigenetic changes in monozygotic twins discordant for schizophrenia and bipolar disorder. Hum. Mol. Genet. 20, 4786-4796 (2011).

16. Lahiri, D. K, Maloney, B. \& Zawia, N. H. The LEARn model: an epigenetic explanation for idiopathic neurobiological diseases. Mol. Psychiatry 14, 992-1003 (2009).

17. Khaitovich, P. et al. Regional patterns of gene expression in human and chimpanzee brains. Genome Res. 14, 1462-1473 (2004).

18. Ladd-Acosta, C. et al. DNA methylation signatures within the human brain. Am. J. Hum. Genet. 81, 1304-1315 (2007). 
19. Johnston-Wilson, N. L. et al. Disease-specific alterations in frontal cortex brain proteins in schizophrenia, bipolar disorder, and major depressive disorder. The Stanley Neuropathology Consortium. Mol. Psychiatry 5, 142-149 (2000).

20. Cechetto, D. F., Weishaupt, N. The Cerebral Cortex in Neurodegenerative and Neuropsychiatric Disorders: Experimental Approaches to Clinical Issues.

21. Cheng, W. Y., Ou Yang, T. H. \& Anastassiou, D. Biomolecular events in cancer revealed by attractor metagenes. PLoS Comput. Biol. 9, e1002920 (2013).

22. Gautier, L., Cope, L., Bolstad, B. M. \& Irizarry, R. A. Affy-analysis of affymetrix GeneChip data at the probe level. Bioinformatics 20,307-315 (2004).

23. Pidsley, R. et al.A data-driven approach to preprocessing Illumina 450K methylation array data. BMC Genom. 14, 293 (2013).

24. Hokama, M. et al. Altered expression of diabetes-related genes in Alzheimer's disease brains: the Hisayama Study. Cereb. Cortex 24, 2476-2488 (2014).

25. $\mathrm{Xu}, \mathrm{H}$. et al. Sex-biased methylome and transcriptome in human prefrontal cortex. Hum. Mol. Genet. 23, 1260-1270 (2014).

26. Dumitriu, A. et al. Integrative analyses of proteomics and RNA transcriptomics implicate mitochondrial processes, protein folding pathways and GWAS loci in Parkinson disease. BMC Med. Genomics. 9, 5 (2016).

27. Pantazatos, S. P. et al. Whole-transcriptome brain expression and exon-usage profiling in major depression and suicide: evidence for altered glial, endothelial and ATPase activity. Mol. Psychiatr. 22, 760-773 (2017).

28. Akbarian, S. et al. The PsychENCODE project. Nat. Neurosci. 18, 1707-1712 (2015).

29. Love, M. I., Huber, W. \& Anders, S. Moderated estimation of fold change and dispersion for RNA-seq data with DESeq2. Genome Biol. 15, 550 (2014).

30. Cheng, W.-Y., Yang, T.-H. O., Shen, H., Laird, P. W., Anastassiou, D. Cancer Genome Atlas Research Network t (2013). Multi-cancer molecular signatures and their interrelationships. In: ArXiv e-prints.

31. Cover T. M., Thomas J. A. Elements of information theory, 2nd edn. WileyInterscience: Hoboken, N.J., 2006, xxiii, p. 748.

32. Du, P. et al. Comparison of Beta-value and M-value methods for quantifying methylation levels by microarray analysis. BMC Bioinforma. 11, 587 (2010).

33. Subramanian, A. et al. Gene set enrichment analysis: a knowledge-based approach for interpreting genome-wide expression profiles. Proc. Natl Acad. Sci. USA 102, 15545-15550 (2005).

34. Szklarczyk, D. et al. The STRING database in 2017: quality-controlled protein-protein association networks, made broadly accessible. Nucleic Acids Res. 45(D1), D362-D368 (2017).

35. Bates, D., Machler, M., Bolker, B. M. \& Walker, S. C. Fitting linear mixed-effects models using Ime4. J. Stat. Softw. 67, 1-48 (2015).

36. Luke, S. G. Evaluating significance in linear mixed-effects models in R. Behav. Res. Methods 49, 1494-1502 (2017).

37. Bangasser, D. A. et al. Corticotropin-releasing factor overexpression gives rise to sex differences in Alzheimer's disease-related signaling. Mol. Psychiatry 22, 1126-1133 (2017).

38. Avner, P. \& Heard, E. X-chromosome inactivation: counting, choice and initiation. Nat. Rev. Genet. 2, 59-67 (2001).

39. Darmanis, S. et al. A survey of human brain transcriptome diversity at the single cell level. Proc. Natl Acad. Sci. USA 112, 7285-7290 (2015).

40. Zeisel, A. et al. Brain structure. Cell types in the mouse cortex and hippocampus revealed by single-cell RNA-seq. Science 347, 1138-1142 (2015).

41. Guintivano, J., Aryee, M. J. \& Kaminsky, Z. A. A cell epigenotype specific model for the correction of brain cellular heterogeneity bias and its application to age, brain region and major depression. Epigenetics 8, 290-302 (2013).

42. Blalock, E. M. et al. Incipient Alzheimer's disease: microarray correlation analyses reveal major transcriptional and tumor suppressor responses. Proc. Natl Acad. Sci. USA 101, 2173-2178 (2004).

43. Gandal, M. J. et al. Shared molecular neuropathology across major psychiatric disorders parallels polygenic overlap. Science 359, 693-697 (2018).
44. Mistry, M., Gillis, J. \& Pavlidis, P. Genome-wide expression profiling of schizophrenia using a large combined cohort. Mol. Psychiatry 18, 215-225 (2013).

45. Kim, S. \& Webster, M. J. Correlation analysis between genome-wide expression profiles and cytoarchitectural abnormalities in the prefrontal cortex of psychiatric disorders. Mol. Psychiatry 15, 326-336 (2010).

46. Finn, R. D. et al. InterPro in 2017-beyond protein family and domain annotations. Nucleic Acids Res. 45(D1), D190-D199 (2017).

47. Catts, V. S., Wong, J., Fillman, S. G., Fung, S. J. \& Shannon Weickert, C. Increased expression of astrocyte markers in schizophrenia: association with neuroinflammation. Aust. N. Z. J. Psychiatry 48, 722-734 (2014).

48. Gonzalez-Reyes, R. E., Nava-Mesa, M. O., Vargas-Sanchez, K., Ariza-Salamanca, D. \& Mora-Munoz, L. Involvement of Astrocytes in Alzheimer's Disease from a neuroinflammatory and oxidative stress perspective. Front. Mol. Neurosci. 10, 427 (2017).

49. Rial, D. et al. Depression as a glial-based synaptic dysfunction. Front. Cell Neurosci. 9, 521 (2016).

50. Sun, J. D., Liu, Y., Yuan, Y. H., Li, J. \& Chen, N. H. Gap junction dysfunction in the prefrontal cortex induces depressive-like behaviors in rats. Neuropsychopharmacology 37, 1305-1320 (2012).

51. Briere, F. N., Rohde, P., Seeley, J. R., Klein, D. \& Lewinsohn, P. M. Comorbidity between major depression and alcohol use disorder from adolescence to adulthood. Compr. Psychiatry 55, 526-533 (2014).

52. Sullivan, P. F., Neale, M. C. \& Kendler, K. S. Genetic epidemiology of major depression: review and meta-analysis. Am. J. Psychiatry 157, 1552-1562 (2000).

53. Fillman, S. G., Sinclair, D., Fung, S. J., Webster, M. J. \& Shannon Weickert, C. Markers of inflammation and stress distinguish subsets of individuals with schizophrenia and bipolar disorder. Transl. Psychiatry 4, e365 (2014).

54. Stankiewicz, A. M., Swiergiel, A. H. \& Lisowski, P. Epigenetics of stress adaptations in the brain. Brain Res. Bull. 98, 76-92 (2013).

55. Ryan, M. M. et al. Gene expression analysis of bipolar disorder reveals downregulation of the ubiquitin cycle and alterations in synaptic genes. Mol. Psychiatry 11, 965-978 (2006).

56. Maycox, P. R. et al. Analysis of gene expression in two large schizophrenia cohorts identifies multiple changes associated with nerve terminal function. Mol. Psychiatry 14, 1083-1094 (2009).

57. Zhang, Y., James, M., Middleton, F. A. \& Davis, R. L. Transcriptional analysis of multiple brain regions in Parkinson's disease supports the involvement of specific protein processing, energy metabolism, and signaling pathways, and suggests novel disease mechanisms. Am. J. Med. Genet. B Neuropsychiatr. Genet. 137B, 5-16 (2005).

58. Zheng, B. et al. PGC-1alpha, a potential therapeutic target for early intervention in Parkinson's disease. Sci. Transl. Med 2, 52 ra73 (2010).

59. Narayan, S. et al. Molecular profiles of schizophrenia in the CNS at different stages of illness. Brain Res. 1239, 235-248 (2008).

60. Chang, L. C. et al. A conserved BDNF, glutamate- and GABA-enriched gene module related to human depression identified by coexpression metaanalysis and DNA variant genome-wide association studies. PLoS One $\mathbf{9}$, e90980 (2014).

61. Lunnon, K. et al. Methylomic profiling implicates cortical deregulation of ANK1 in Alzheimer's disease. Nat. Neurosci. 17, 1164-1170 (2014).

62. Wockner, L. F. et al. Genome-wide DNA methylation analysis of human brain tissue from schizophrenia patients. Transl. Psychiatry 4, e339 (2014).

63. Pidsley, R. et al. Methylomic profiling of human brain tissue supports a neurodevelopmental origin for schizophrenia. Genome Biol. 15, 483 (2014).

64. Jaffe, A. E. et al. Mapping DNA methylation across development, genotype and schizophrenia in the human frontal cortex. Nat. Neurosci. 19, 40-47 (2016).

65. Smith, R. G. et al. Elevated DNA methylation across a 48-kb region spanning the HOXA gene cluster is associated with Alzheimer's disease neuropathology. Alzheimers Dement. 14, 1580-1588 (2018). 